\title{
Avaliação do Uso de Técnicas PIR-G/FT-IR para Caracterização de Elastômeros
}

\author{
Natália B. Sanches \\ Instituto Tecnológico da Aeronáutica e Centro Logístico da Aeronáutica, CELOG, SP \\ Milton F. Diniz, Tiago B. Reis, Silvana N. Cassu, Rita C. L. Dutra \\ Divisão de Química, CTA, SP
}

Resumo: A técnica de pirólise gasosa, em bico de Bunsen, para análise por espectroscopia no infravermelho com transformada de Fourier (PIR-G/FT-IR) foi aplicada a diferentes borrachas, incluindo algumas misturas. Foi observado que é possível diferenciar os tipos de elastômeros por meio de análise de produtos gasosos de pirólise, inclusive aqueles que apresentam espectros IR de pirolisados líquidos similares, como é o caso de CIIR e BIIR, NR/SBR e EPDM/SBR, SBR/ BR e SBR.

Palavras-chave: PIR-G/FT-IR, borrachas ou elastômeros, NBR, EPDM, SBR, NR, BR, CSM, IIR, CIIR, BIIR, Viton, MQ.

\section{Evaluation of PIR-G/FT-IR techniques for characterization of elastomers}

Abstract: Pyrolysis and infrared spectroscopy (PIR-G/FT-IR) were used for investigating gaseous products of rubber. The results show that this method was suitable to identify different elastomers and elastomer blends, including rubbers that present similar IR spectra of pyrolysed liquid products such as CIIR and BIIR, NR/SBR and EPDM/SBR, SBR/BR and SBR.

Keywords: PIR-G/FT-IR, rubber or elastomer, NBR, EPDM, SBR, NR, BR, CSM, IIR, CIIR, BIIR, Viton, MQ.

\section{Introdução}

É conhecido que borrachas cruas ou vulcanizadas são adequadamente analisadas por meio de técnicas espectroscópicas quando pirolisadas, ou seja, após serem submetidas à degradação térmica ${ }^{[1-3]}$.

A espectroscopia no infravermelho (IR) dispersiva ou com transformada de Fourier (FT-IR) pode ser utilizada para a identificação inequívoca da maioria dos elastômeros e suas misturas por meio da análise dos espectros FT-IR de seus pirolisados líquidos, bem como para sua quantificação ${ }^{[4-7]}$. Entretanto, existem algumas composições elastoméricas que apresentam espectros FT-IR similares aos seus pirolisados líquidos e a investigação de seus produtos gasosos pode constituir uma importante ferramenta para a diferenciação destes tipos de borrachas ${ }^{[8]}$.

Pattacini ${ }^{[8]}$, embora tenha analisado um pequeno número de tipos de borracha, poli (cis-isopreno) (NR), policloropreno (CR), policlorossulfoetileno (CSM) e polibutadieno (BR), mostrou que as absorções IR dos pirolisados gasosos de BR e CSM podem ser usadas para diferenciar esses tipos de elastômeros, os quais apresentam similaridade entre os espectros de seus pirolisados líquidos.

Há também interesse na investigação dos produtos gasosos de pirólise em relação à segurança relativa à concen- tração de produtos tóxicos em laboratórios e ambientes de processo ou desenvolvimento de materiais.

Deste modo, o desenvolvimento de metodologias simples e rápidas, que permitam a identificação dessas composições, torna-se atrativo para pesquisas realizadas em diferentes áreas industriais que trabalhem com elastômeros.

É conhecido que o uso de acoplamento de técnicas instrumentais, como análise termogravimétrica/infravermelho (TG/ FT-IR) cromatografia gasosa/IR (CG/IR) e CG/espectrometria de massa (MS) tem sido útil para diversas pesquisas envolvendo caracterização de elastômeros, entre outros polí$\operatorname{meros}^{[9-11]}$. Entretanto, existem algumas desvantagens no uso dessas metodologias, como custo de equipamentos e análises. Portanto, métodos que envolvam acessórios mais simples, de uso comum nos diferentes laboratórios, são extremamente úteis e despontam como metodologias alternativas.

Desde que o uso da espectroscopia IR para caracterização de elastômeros tem apresentado bons resultados em nossos laboratórios ${ }^{[6,7,12-14]}$ e diante da importância de pesquisas desenvolvidas na área de elastômeros, materiais utilizados em várias aplicações, entre elas a indústria aeroespacial, de interesse nacional, o presente trabalho tem por objetivo apresentar a avaliação da aplicabilidade da técnica PIR-G/FT-IR como método simples, rápido e de baixo custo para a caracterização de diferentes tipos de elastômeros.

Autor para correspondência: Rita C. L. Dutra, Divisão de Química, IAE, CTA, Praça Mal. Eduardo Gomes 50, CEP: 12228-904, São José dos Campos, SP. E-mail: ritad@iae.cta.br 
Tabela 1. Elastômeros utilizados e seus respectivos fornecedores.

\begin{tabular}{|c|c|c|}
\hline Elastômero & Sigla & Fornecedor \\
\hline Poli (butadieno-co-acrilonitrila) & NBR & $\mathrm{EBO} *$ \\
\hline $\begin{array}{l}\text { Poli(etileno-co-propileno-co dieno } \\
\text { ou polieno) }\end{array}$ & EPDM & $\mathrm{EBO}^{*}$ \\
\hline Poli(butadieno-co-estireno) & SBR & $\mathrm{EBO}^{*}$ \\
\hline Borracha natural & NR & EBO* \\
\hline Borracha butílica & BR & $\mathrm{EBO}^{*}$ \\
\hline Poli(clorosulfoetileno) (Hypalon) & CSM & Pagé \\
\hline Poli(isobutileno-co-isopreno) & IIR & Nifriflex \\
\hline Borracha clorobutílica & CIIR & Parabor \\
\hline Borracha bromobutílica & BIIR & Parabor \\
\hline $\begin{array}{l}\text { Poli(hexafluorpropileno-co-fluoreto } \\
\text { de vinilideno) }\end{array}$ & Viton & Du Pont \\
\hline $\begin{array}{l}\text { Poli(siloxano) (borracha de } \\
\text { silicone) }\end{array}$ & MQ & Pagé \\
\hline
\end{tabular}

* Borrachas preparadas em nosso Centro de Pesquisas, contendo componentes utilizados em formulações típicas para essas borrachas.

\section{Experimental}

Os elastômeros utilizados nesse estudo, bem como seus fornecedores são mostrados na Tabela 1 .

As amostras vulcanizadas foram usadas tal como recebidas. Entretanto, para teste da metodologia, a amostra de BIIR foi extraída em acetona e pirolisada, antes e após extração, com e sem controle de tempo e temperatura. As temperaturas utilizadas foram 500 e $700^{\circ} \mathrm{C}$ e o tempo 1 e 3 min.

A Figura 1 mostra o dispositivo usado para obtenção de pirolisados gasosos, baseado na metodologia desenvolvida por Pattacini ${ }^{[8]}$. Na realização do ensaio, uma quantidade de amostra entre 0,5 e $1,5 \mathrm{~g}$, em pequenos pedaços, é colocada em tubo de pirólise e aquecida rapidamente em bico de Bunsen. O tubo de pirólise, conforme mostra a Figura 1, é conectado por meio de tubo de silicone a uma celula de gás previamente aquecida $\left(50^{\circ} \mathrm{C}\right.$ por 15 min para manter a célula em condições adequadas à análise da fase gasosa do pirolisado obtido). O tempo de pirólise é o necessário para preencher a célula de gás.

Os pirolisados foram imediatamente analisados, na forma gasosa, no espectrômetro FT-IR SPECTRUM 2000 PERKIN ELMER (resolução $4 \mathrm{~cm}^{-1}$, ganho $1,4000 \mathrm{a} 400 \mathrm{~cm}^{-1}$, 1 varredura) em célula para análise de gás (10 $\mathrm{cm}$ de caminho ótico).

\section{Resultados e Discussão}

\section{Análise por FT-IR dos produtos gasosos de pirólise de borrachas}

$\mathrm{Na}$ literatura ${ }^{[16]}$, os espectros dos pirolisados líquidos dos elastômeros são mais divulgados do que os pirolisados gasosos correspondentes. Deste modo, a Figura 2 inclui os espectros FTIR obtidos em nossos laboratórios, para os produtos gasosos de

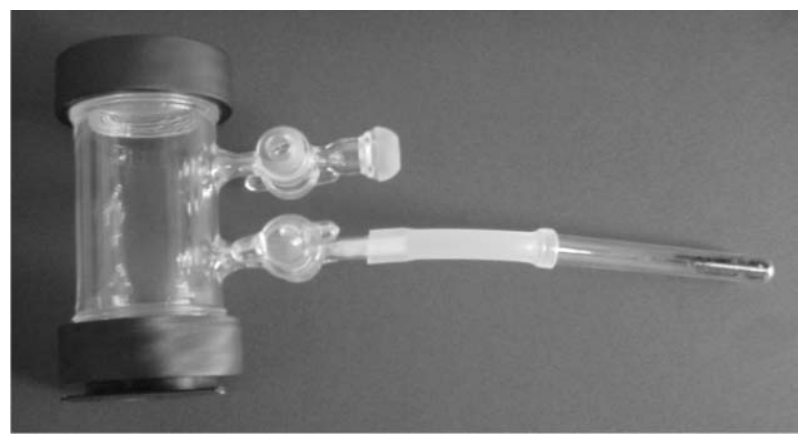

Figura 1. Dispositivo utilizado para a obtenção de pirolisados gasosos

pirólise dos elastômeros mais usuais. Pode ser observado que existem regiões, contendo bandas características, que permitem a diferenciação das borrachas mostradas na Figura 2.

Na Figura 2 observa-se que NR pode ser diferenciada das demais borrachas pelas absorções fortes em 1603 e $905 \mathrm{~cm}^{-1}$, que estão associadas à presença de grupos $\mathrm{C}=\mathrm{C}^{[17]}$, presentes em isopreno, principal componente do pirolisado gasoso de $\mathrm{NR}$, segundo a literatura ${ }^{[16 a]}$. BR apresenta banda intensa na região próxima a $1600 \mathrm{~cm}^{-1}$, que está associada aos grupos $\mathrm{C}=\mathrm{C}^{[10,11]}$ presentes em compostos à base de butadieno. No caso específico do pirolisado de BR, 1,3 polibutadieno, seu principal componente ${ }^{[16 b]}$ também o diferencia dos outros tipos de borracha, principalmente do Hypalon, que apresenta similaridade quando são comparados somente os espectros FT-IR dos pirolisados líquidos ${ }^{[8]}$. O espectro do pirolisado gasoso do Hypalon mostra ainda absorções na região entre $2500-3000 \mathrm{~cm}^{-1}$, as quais provavelmente devem estar associadas à presença de $\mathrm{HCl}^{[10]}$.

Viton pode ser identificado por meio das regiões 1000-

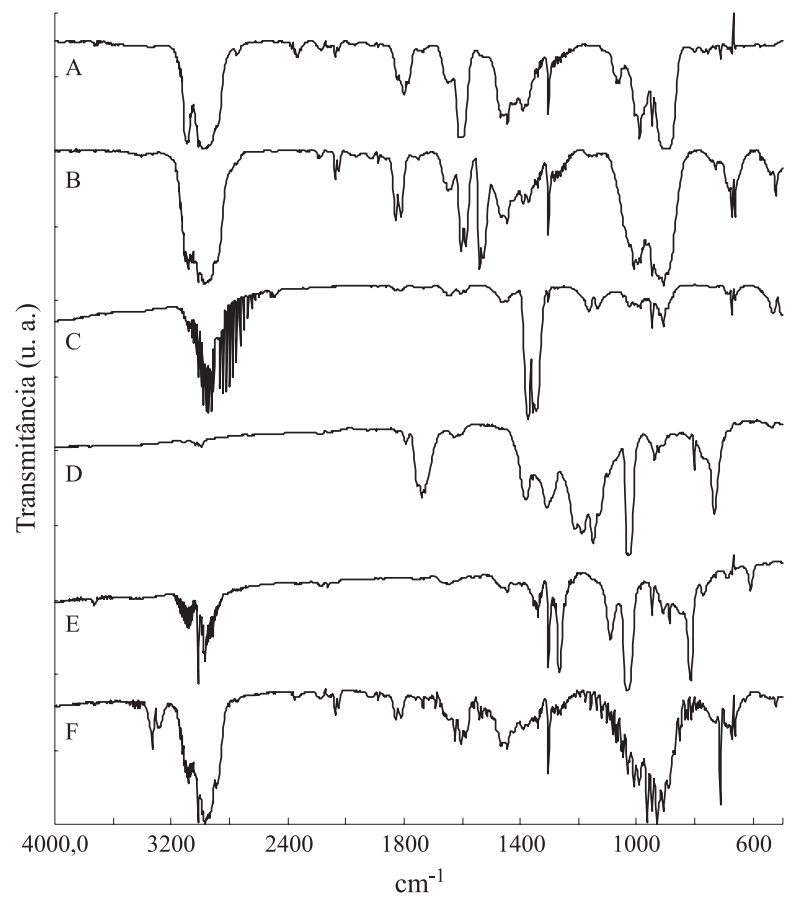

Figura 2. Espectros FT-IR, obtidos em nossos laboratórios, da fase gasosa dos pirolisados de borrachas: (A) NR, (B) BR, (C) Hypalon, (D) Viton, (E) Silicone e (F) NBR. 
$1100 \mathrm{~cm}^{-1}$ e $733 \mathrm{~cm}^{-1}$ (grupo C-F), características de compostos fluorados ${ }^{[12]}$.

Borracha de silicone apresenta bandas intensas em $1264 \mathrm{e}$ $817 \mathrm{~cm}^{-1}$ (grupos Si-C) ${ }^{[11 \mathrm{c}]}$.

Um destaque é feito nesse trabalho para a borracha NBR, que é utilizada como isolante térmico em motor-foguete ${ }^{[19]}$, e portanto, de maior interesse para as atividades desenvolvidas em nosso Centro de Pesquisa. $\mathrm{O}$ espectro do pirolisado gasoso da NBR pode ser diferenciado dos demais por apresentar bandas em $2176 \mathrm{~cm}^{-1}$, que provavelmente caracteriza a presença de HCN, e bandas localizadas em 992, 965 e 908 $\mathrm{cm}^{-1}$ que devem estar relacionadas com a presença de ligações $\mathrm{C}=\mathrm{C}$, o que confirma dados da literatura ${ }^{[17]}$.

Embora a literatura ${ }^{[17]}$ não cite a atribuição dessas bandas,
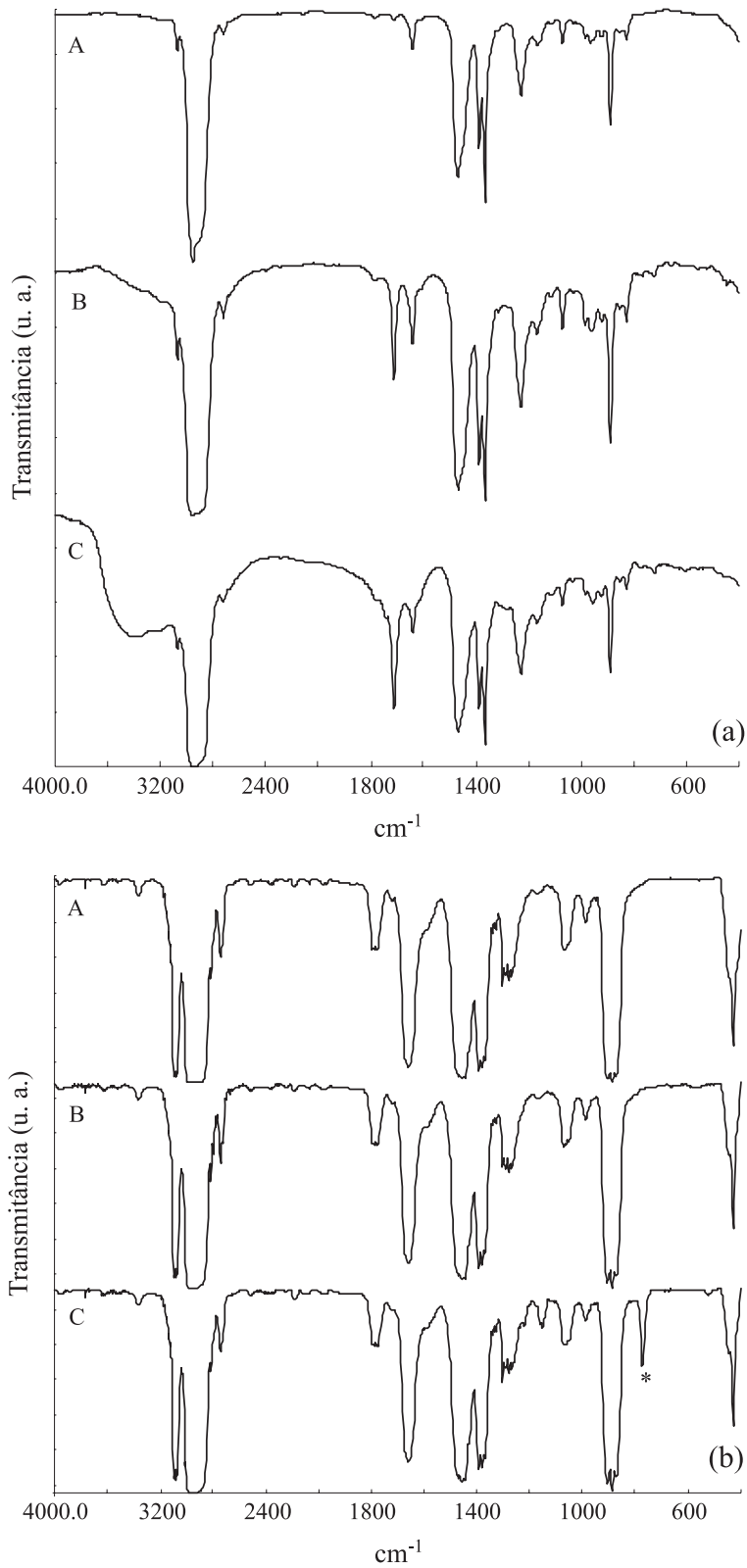

Figura 3. (a) Espectros FT-IR de pirolisados líquidos de (A) IIR, (B) CIIR e (C) BIIR; (b) Espectros FT-IR de pirolisados gasosos de (A) IIR, (B) CIIR e (C) BIIR.

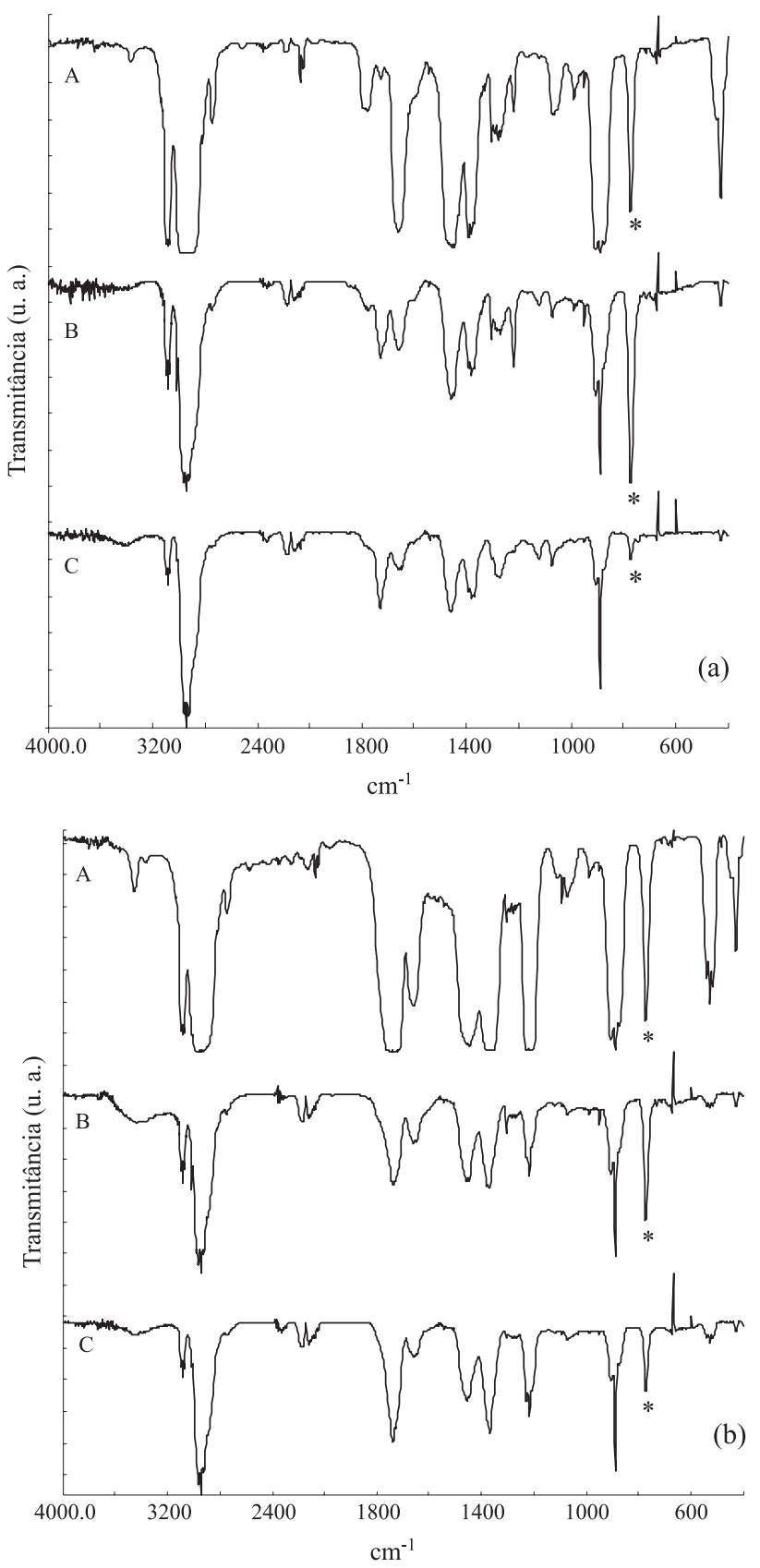

Figura 4. (a) Espectros FT-IR obtidos para BIIR sem extração: A) pirolisado gasoso obtido em bico de Bunsen; B) pirolisado gasoso obtido em pirolisador a $700{ }^{\circ} \mathrm{C} / 1 \mathrm{~min}$ e C) pirolisado gasoso obtido em pirolisador a $500^{\circ} \mathrm{C} / 3 \mathrm{~min}$; (b) Espectros FT-IR obtidos para BIIR após extração em acetona: A) pirolisado gasoso obtido em bico de Bunsen; B) pirolisado gasoso obtido em pirolisador a $700{ }^{\circ} \mathrm{C} / 1 \mathrm{~min}$ e C) pirolisado gasoso obtido em pirolisador a $500{ }^{\circ} \mathrm{C} / 3 \mathrm{~min}$.

comenta que o elastômero NBR apresenta, além de HCN, eteno e butadieno como produtos gasosos de pirólise.

Borrachas com espectros IR de pirolisados líquidos similares/Diferenciação por meio de pirolisados gasosos

\section{IIR/CIIR/BIIR}

A borracha IIR e suas formas halogenadas, CIIR e BIIR apresentam espectros IR de pirolisados líquidos similares 
(Figura 3-a). Entretanto, por meio da análise dos produtos gasosos de pirólise em bico de Bunsen pode ser estabelecida uma diferença entre a BIIR e CIIR por meio da banda em $772 \mathrm{~cm}^{-1}$ presente no espectro da BIIR, provavelmente atribuída ao grupo C-Br (Figura 3-b).

Para testar a metodologia descrita, ou seja, avaliar a validade da pirólise gasosa em bico de Bunsen, BIIR foi escolhida. A amostra foi pirolisada com e sem extração em acetona, em um pirolisador com controle de temperatura e tempo $\left(\right.$ a $500^{\circ} \mathrm{C}$ por 3 minutos e a $700{ }^{\circ} \mathrm{C}$ por 1 minuto). As Figuras 4-a e 4-b incluem os espectros resultantes.

Os resultados apresentados nas Figuras 4-a e 4-b mostram que independente da amostra ser extraída ou não, pirolisada com ou sem controle de temperatura e tempo, apresenta a banda em $772 \mathrm{~cm}^{-1}$, que a diferencia de CIIR e IIR. Esse resultado mostra que a metodologia de pirólise em bico de Bunsen desenvolvida em nossos laboratórios, pode ser utilizada para a diferenciação de borrachas que apresentam espectros de pirolisados líquidos semelhantes, validando a metodologia proposta. A utilização desse método permite a redução do tempo e do custo de análise por meio de dispositivos mais simples do que um pirolisador com tempo e temperatura controlados, e sem a necessidade de extração da borracha.

\section{$N R / S B R$ e $E P D M / S B R$}

Os espectros IR dos pirolisados líquidos de mistura de borrachas NR/SBR mostram similaridade com os correspondentes da mistura EPDM/SBR, sendo observadas principalmente as absorções relativas ao SBR (Figura 5-a), conforme mostrado em trabalho anterior ${ }^{[19]}$. Contudo, os pirolisados gasosos dessas duas misturas apresentam espectros diferentes (Figura 5-b). O espectro correspondente à mistura NR/SBR 50:50 (A), por exemplo, mostra mais evidentemente as bandas de NR (B), enquanto no espectro da mistura EPDM/SBR 50:50 (C), as bandas relativas ao SBR (D) são mais nítidas, acrescidas de algumas poucas absorções de $\operatorname{EPDM}(\mathrm{E})$, entre 950 e $1000 \mathrm{~cm}^{-1}$, provavelmente atribuídas aos grupos $\mathrm{C}=\mathrm{C}$. Existe ainda uma banda em $772 \mathrm{~cm}^{-1}$, que pode estar associada aos grupos aromáticos do estireno presente no SBR.

\section{$B R / S B R$}

Nas Figuras 6-a e 6-b encontram-se, respectivamente, os espectros dos pirolisados líquidos e gasosos obtidos para a mistura SBR/BR 50:50 e para os elastômeros individuais. O espectro do pirolisado líquido de SBR/BR apresenta similaridade com o espectro de SBR (Figura 6-a) sendo que as absorções da borracha BR são sobrepostas pelas absorções do copolímero SBR, dificultando a diferenciação entre a borracha SBR pura e uma mistura SBR/BR. No entanto, o pirolisado gasoso de SBR/BR 50:50 apresenta absorção em $1600 \mathrm{~cm}^{-1}$, característica de BR (Figura 6-b). Neste caso, a associação dos espectros FT-IR obtidos pela análise de pirolisados líquido e gasoso permite a diferenciação entre SBR ou BR e uma mistura SBR/BR.

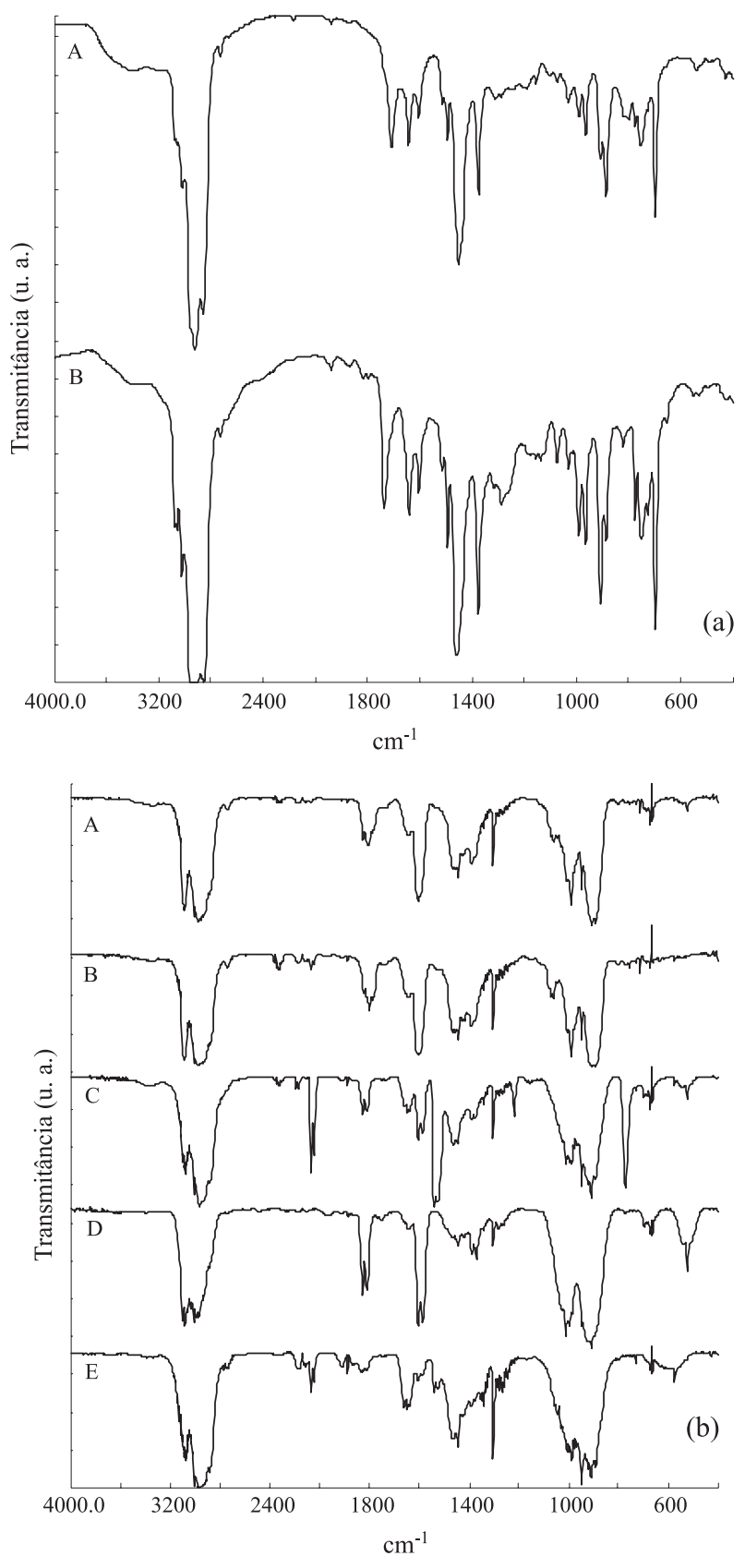

Figura 5. (a) Espectros FT-IR de pirolisados líquidos de (A) NR/SBR 50:50 e (B) EPDM/SBR 50:50; (b) Espectros FT-IR de pirolisados gasosos de: (A) NR/SBR 50:50, (B) NR, (C) EPDM/SBR 50:50, (D) SBR e (E) EPDM.

\section{Misturas contendo NR}

É interessante observar que, quando se comparam espectros dos pirolisados gasosos de NR/SBR 20:80 e NR/SBR 50:50, as bandas de SBR são mais evidenciadas como esperado no primeiro espectro (Figura 7-a), e as bandas de NR em razão de alguma sobreposição não são tão facilmente detectadas. O mesmo acontece com CR/NR 80:20, ou seja, as bandas de CR são mais evidenciadas (Figura 7-b), sugerindo que provavelmente, pode ser feita uma avaliação semi-quantitativa do teor de produtos gasosos de pirólise em misturas de borrachas, entretanto, com ressalvas para teores iguais ou inferiores a $20 \mathrm{phr}$. 

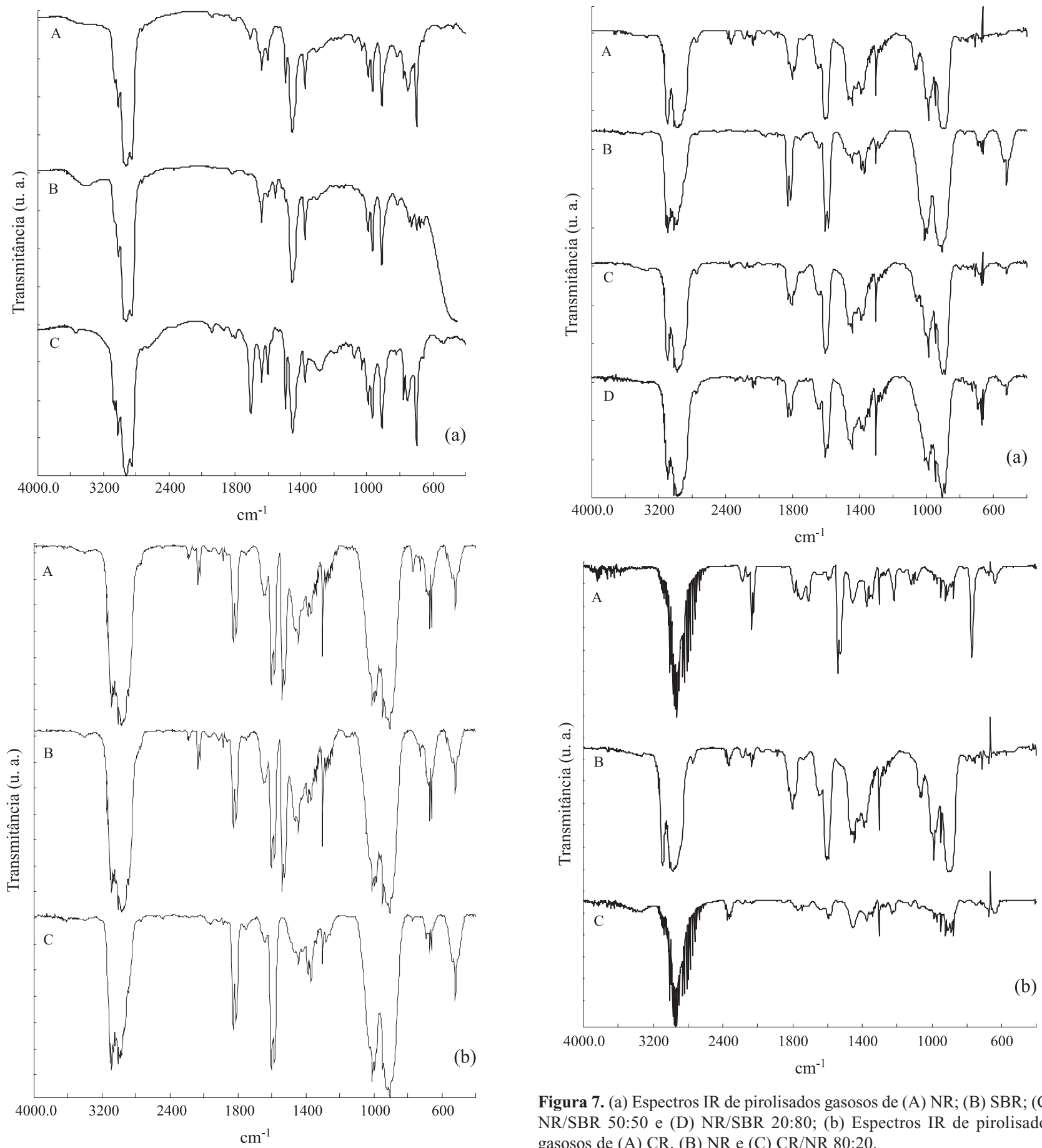

Figura 7. (a) Espectros IR de pirolisados gasosos de (A) NR; (B) SBR; (C) NR/SBR 50:50 e (D) NR/SBR 20:80; (b) Espectros IR de pirolisados gasosos de (A) CR, (B) NR e (C) CR/NR 80:20.

Figura 6. (a) Espectros IR de pirolisados líquidos de (A) SBR/BR 50:50, (B) BR e (C) SBR; (b) Espectros IR de pirolisados gasosos de (A) SBR/BR 50:50, (B) BR e (C) SBR.

Deve ser também destacado, que na literatura ${ }^{[16]}$, não foram encontrados espectros dos pirolisados gasosos de Viton e misturas de borrachas, nem as atribuições das bandas relativas aos grupos presentes em borrachas mais usuais, tais como NBR, SBR e NR. Portanto, a inclusão, nesse trabalho, dos espectros dos pirolisados gasosos das citadas borrachas e a atribuição das suas bandas características, assim como das borrachas mais usadas na indústria, constituem uma contribuição à pesquisa de caracterização de elastômeros.

\section{Conclusão}

A aplicação de PIR-G/FT-IR para caracterização de elastômeros, dentro dos limites de detecção da técnica, sugeriu que:

- é possível diferenciar elastômeros por meio de análise de produtos gasosos de pirólise, inclusive aqueles que apresentam espectros IR de pirolisados líquidos similares, tais como CIIR e BIIR, NR/SBR 50:50 e EPDM/SBR 50:50, BR e $\mathrm{SBR} / \mathrm{BR}$ e $\mathrm{SBR}$.

- o método de identificação de elastômeros pela utilização 
de análise FT-IR de pirolisado gasoso obtido em bico de Bunsen foi verificado em borracha BIIR, observando-se que o espectro IR de seu pirolisado gasoso apresenta a banda em $772 \mathrm{~cm}^{-1}$, que a diferencia de CIIR e IIR, independente da amostra ser ou não extraída e obtida em pirolisador com cintrole de temperatura e tempo ou em bico de Bunsen.

- há indícios que pode ser feita uma avaliação semi-quantitativa do teor de produtos gasosos de pirólise de misturas de borrachas, entretanto, com ressalvas para teores iguais ou inferiores a $20 \mathrm{phr}$.

\section{Referências Bibliográficas}

1. Matheson, J. M.; Wampler, T. P. \& Simonsick, W. J. - Journal of Analytical and Applied Pyrolysis, 29, p.129 (1994).

2. Wake, W. C.; Tidd, B. K. \& Loadman, M. J. R. - "Analysis of Rubber and Rubber-like Polymers", third Edition, Applied Science Publishers (1983).

3. Williams, P. T. \& Besler, S. - Fuel, 74 (9), p.1277, (1995).

4. Gedeon, B. J. \& Ngyuen, R. H. - Rubber Division Meeting, American Chemical Society, Cleveland, Ohio, paper 64 Oct, p.1, (1985).

5. Annual Book of ASTM Standards, Part 37 ASTM D3677 (1982).

6. Dutra, R. C. L. -Analyse quantitative infrarouge des associations du caoutchouc, Publicação interna IFOCA, Paris, (1988).

7. Dutra, R. C. L.; Diniz, M .F.;. Ribeiro, A. P.; Lourenço, V. L.; Cassu, S. N. \& Azevedo, M. F. P. - Polímeros: Ciência e Tecnologia, 14 (5), p.334 (2004).
8. Pattacini, S. C. - Perkin-Elmer Infrared Bulletin, october, order n. IRB-52 (1975).

9. McGrattan, Brian. - Journal of Applied Spectroscopy 48 (12), p.1472 (1994).

10. Hiltz, J. A. - Journal of Analytical and Applied Pyrolysis, 55, p.135, (2000).

11. Ghebremeskel, G. N.; Sekinger, J. K.; Hoffauir \& J. L.; Hendrix, C. - Rubber Chemistry Technology, 69 (5), p.874, (1996).

12. Dutra, R. C. L.; Diniz, M. F. \& Lourenço, V. L.- II ABPOL - SP II ABPOL - SP - (1993).

13. Dutra, R. C. L. - Polímeros: Ciência e Tecnologia; p.26 (1996).

14. Dutra, R. C. L.; Dutra, J. C. N.; Massi, M.; Otani, C.; Urruchi, W. I.; Maciel, H. S.; Diniz, M. F. \& Bittencourt, E. Molecular Crystals and Liquid Crystals; 374, p.45 (2002).

15. Babbit, R. O. - "The Vanderbilt Rubber handbook", R.T. Vanderbilt Company Inc., Norwalk (1978).

16. School, F. - "Atlas: Polymer and Plastics Analysis", Verlag Chemie, vol III, a) p.3700, b) p.3691, c) p.3853 (1981).

17. Smith, A. L. - Applied Infrared Spectroscopy, John Wiley \& Sons, New York, (1979).

18. Sayles, D. C. - Rubber Chem. Techn., 39, p.112 (1966).

19. Dutra, R. C. L. \& Diniz, M. F. - Polímeros: Ciência e Tecnologia, Julho/Setembro p.25 (1993).

Enviado: 28/06/05

Reenviado: 10/10/05

Aprovado: 01/03/06 